Check for updates

Cite this: RSC Adv., 2017, 7, 52449

\title{
Electronic structure and optical properties of Csl under high pressure: a first-principles study
}

\begin{abstract}
Qiang Zhao, (D) *a Zheng Zhang ${ }^{\text {a }}$ and Xiaoping Ouyang ${ }^{\text {abc }}$
Cesium iodide (Csl) is an important scintillation material and it is widely used in high-energy physics experiments, medical imaging, security inspection and other fields as a key irradiation sensor. The detection efficiency of $\mathrm{Csl}$ depends on its electronic structure and optical properties. High pressure and doping are the two most commonly used methods which can regulate and control the electronic structure and optical properties of an ionic crystal material. In this paper, we investigated the effects of high pressure on the electronic structure and optical properties of a Csl crystal through a first-principles calculation method based on density functional theory, and the exchange and correlation functions among electrons were described using the revised Perdew-Burke-Ernzerhof generalized gradient approximation. The band gap of a Csl single crystal decreases with an increase in pressure; every part of the valence band becomes wider and wider, the conduction band moves in the direction of decreasing energy; the atomic charge transfers from Cs atoms to I atoms in CsI when the external high pressure is less than $15 \mathrm{GPa}$, on the contrary, the atomic charge transfers from I atoms to Cs atoms in CsI when the high pressure is greater than $15 \mathrm{GPa}$; a redshift occurs in the optical properties of a Csl single crystal under high pressure. Our prediction in this paper might be useful for enhancing the detection efficiency of Csl scintillators.
\end{abstract}

Received 8th August 2017

Accepted 1st November 2017

DOI: 10.1039/c7ra08777b

rsc.li/rsc-advances scintillation detectors such as CsI, the detection efficiency of CsI depends on its electronic structure and optical properties, and high pressure is one of the most commonly used methods which can regulate and control the electronic structure and optical properties of CsI. Therefore, many researchers have studied the effects of high pressure on the structural phase and electronic structure of CsI and similar compounds by experiment. For example, Knittle ${ }^{1}$ and Wang $^{2}$ found that a structural phase transition from a simple cubic phase to a tetragonal phase happened in CsI under a high pressure of about $53 \mathrm{GPa}$ through experimental XRD measurements. Makarenko's ${ }^{3}$ work showed that a redshift occurred in the optical absorption edge of CsI crystals under a pressure of up to $60 \mathrm{GPa}$. $\mathrm{MaO}^{4}$ and Eremets $^{5}$ found that the metallization of CsI crystals happens at a pressure of $115 \mathrm{GPa}$. Gaumé's ${ }^{6}$ research showed that high pressure can enhance the detection of CsI crystals in a high energy range.

With the rapid development of computer technology, the first-principles calculation method is widely used in the research of new kinds of scintillation material. Using the firstprinciples calculation method, $\mathrm{Li}^{7}$ investigated the electronic structure and optical properties of $\mathrm{LaBr}_{3}$ crystals. Ribeiro studied the elastic properties and electronic structure of CsI and its surface. Kurosaki ${ }^{9,10}$ calculated the potential curves for the ground and low-lying excited states of CsI. Liu ${ }^{11}$ studied the optical properties of CsI crystals with a pair of Cs and I vacancies. Ying ${ }^{12}$ found that the perfect CsI crystal does not exhibit an
${ }^{a}$ Beijing Key Laboratory of Passive Safety Technology for Nuclear Energy, North China Electric Power University, Beijing 102206, P. R. China. E-mail: qzhao@ncepu.edu.cn ${ }^{b}$ Northwest Institute of Nuclear Technology, Xi'an 710024, P. R. China

${ }^{c}$ School of Materials Science and Engineering, Xiangtan University, Xiangtan 411105, P. R. China 
absorption band in the visible and near-ultraviolet range while the CsI crystal containing a Cs vacancy exhibits two additional absorption bands which peak at about 2.10 and $3.00 \mathrm{eV}$. As for the effects of high pressure on the crystal structure, electronic structure and optical properties of CsI and similar compounds, Nardelli ${ }^{13}$ found that the cubic-to-tetragonal transition happens at the high-pressure of $44 \mathrm{GPa}$ and $58 \mathrm{GPa}$ for CsI and cesium bromide (CsBr) crystals, respectively. Wei's ${ }^{\mathbf{1 4}}$ research showed that the high-pressure transition phase order is $p m \overline{3} m \rightarrow$ pmma $\rightarrow$ pbam for cesium chloride ( $\mathrm{CsCl}$ ), $p m \overline{3} m \rightarrow$ pmma for $\mathrm{CsBr}$ crystals, and a metallization that was caused by high pressure happened in $\mathrm{CsCl}$ and $\mathrm{CsBr}$. Ullah ${ }^{15}$ found that the band gap of $\mathrm{CsCl}$ decreases with an increase in pressure, and its electronic nature changed from the insulating to metallic at $507 \mathrm{GPa}$. Baghmar $^{16}$ found that the structure of CsI undergoes a transition from $\mathrm{CsCl}\left(\mathrm{B}_{2}\right)$ to tetragonal $\left(\mathrm{B}_{\mathrm{T}}\right)$ and from tetragonal $\left(\mathrm{B}_{\mathrm{T}}\right)$ to orthorhombic $\left(\mathrm{B}_{\mathrm{O}}\right)$ at high pressure. $\mathrm{Xu}^{\prime} \mathrm{s}^{17}$ study showed that the structure phase of the CsI crystal will transfer from the $p m \overline{3} m$ structure to Pmna at $39.5 \mathrm{GPa}$. Although many good pieces of research into the CsI crystal have been completed, as far as we know, a theoretical study of the effects of high pressure on the electronic structure and optical properties of a CsI single crystal is still lacking.

In our past pieces of research, we explored the effects of doping and other inherent defects on the electronic structure and optical properties of $\mathrm{CsI}^{18}$ and $\mathrm{ZnS} .{ }^{19}$ Based on the previous works from other researchers, the main work in this paper is the investigation of the effects of high pressure on the electronic structure and optical properties of CsI. We hope our work might shed some light on the development of CsI as an important scintillation material.

\section{Computational method}

A CsI single crystal has a simple cubic structure, its structure is similar to that of the $\mathrm{CsCl}$ single crystal, and its space group is Pm3m (221). The CsI supercell that we used here consists of 27 Cs atoms and 27 I atoms, and the Cs atom centered in a CsI cell with the I atoms at the corner of the cubic cell. In order to investigate the effects of high pressure on the electronic structure and optical properties of CsI, we applied a series of different equivalent hydrostatic pressures $(5,10,15,20$ and 25 $\mathrm{GPa})$ on the CsI supercell.

Using the first-principles calculation method based on density functional theory (DFT), as implemented in the Cambridge serial total energy packages (CASTEP), ${ }^{20}$ we investigated the electronic structure and optical properties of the CsI crystal under different high pressures. In the crystal periodic potential field, the multi-electron system is expressed by the plane wave function through the three-dimensional periodic boundary condition, in order to reduce the number of plane wave bases and the CASTEP software package uses the ultrasoft pseudopotential to describe the interaction between ions and electrons. The valence-electron configurations of Cs and I atoms are $5 s^{2} 5 p^{6} 6 s^{1}$ and $5 s^{2} 5 p^{5}$, respectively. In reciprocal space, the plane wave cutoff energy was set to $340 \mathrm{eV}$ and the exchange and correlation functions among electrons were described by the revised Perdew-Burke-Ernzerhof (RPBE) ${ }^{\mathbf{2 1}}$ generalized gradient approximation (GGA). The structure optimization was conducted using the Broyden-Fletcher-Goldfarb-Shanno minimization, modified to take into account the total energy as well as the gradients. The self-consistent field (SCF) tolerance was set to $1.0 \times 10^{-6} \mathrm{eV}$ per atom. The $K$-point mesh was set to $5 \times 5 \times 5$ for the electronic structure calculation and optical properties calculation. Optimal atomic positions were determined until satisfying the following conditions: (1) the maximal force on them was less than $0.03 \mathrm{eV} \AA^{-1}$; (2) the maximal change of energy per atom was less than $1.0 \times 10^{-5} \mathrm{eV}$; (3) the maximal displacement was less than $5.0 \times 10^{-4} \AA$; (4) the maximal stress in the supercell was less than $0.02 \mathrm{GPa}$. All calculations were performed on the basis of the optimized lattice structure.

The optical properties of CsI are determined by the frequency-dependent dielectric function $\varepsilon(\omega)=\varepsilon(\omega)+\mathbf{i} \varepsilon_{2}(\omega)$ that mainly connected with the electronic structures. The imaginary part $\varepsilon_{2}(\omega)$ of the dielectric function $\varepsilon(\omega)$ is calculated from the momentum matrix elements between the occupied and unoccupied states with the selection rules. The real part $\varepsilon_{1}(\omega)$ of the dielectric function $\varepsilon(\omega)$ can be derived from the imaginary part $\varepsilon_{2}(\omega)$ using the Kramers-Kronig ${ }^{22}$ dispersion equation. All other optical constants, such as the absorption spectrum, refractive index and reflectivity, can be derived from $\varepsilon_{1}(\omega)$ and $\varepsilon_{2}(\omega)$.

\section{Results and discussion}

\subsection{Structure determination}

Before we investigated the effects of high pressure on the electronic structure and optical properties of CsI, we optimized the geometrical structure of the CsI crystal, and the crystal structure was optimized by force and energy minimization. In the process of optimization, all atoms were moved towards their equilibrium positions. Fig. 1 shows the lattice parameters (after geometrical optimization) and density of CsI under a series of different high pressures, and our results for the lattice constant and density of the CsI crystal at $0 \mathrm{GPa}$ are in good agreement with the results in Nardelli's ${ }^{\mathbf{1 3}}$ research and the values in the

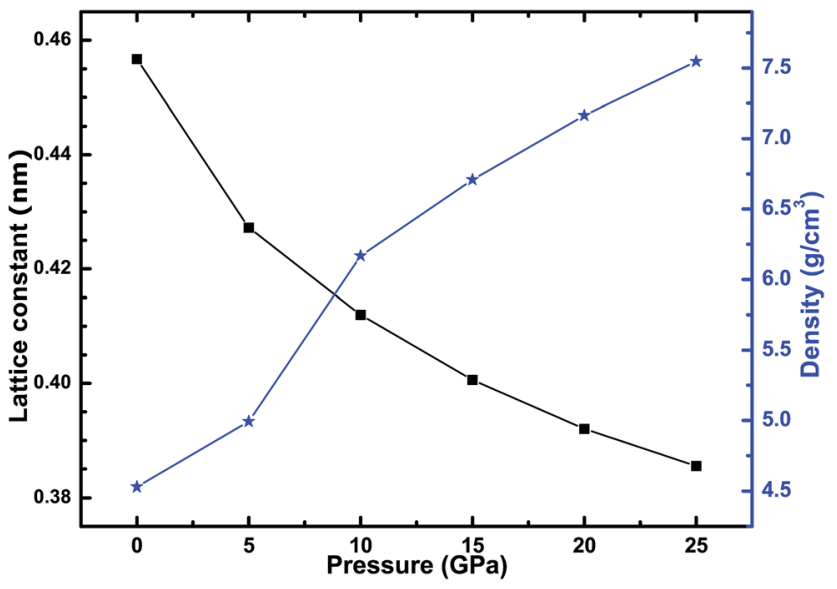

Fig. 1 The lattice constant and density of a Csl cell under high pressure. 
CRC handbook. ${ }^{23}$ The lattice constant of the CsI cell decreases with the increase of the equivalent hydrostatic pressure, while the density of the CsI crystal increases with the equivalent hydrostatic pressure increasing. The reason for these results is that the interaction between atoms in the CsI crystal has changed under the high pressure, as the high pressure makes the bond length between Cs atoms and I atoms become shorter than that of CsI at $0 \mathrm{GPa}$. This change of lattice constant is bound to change the electronic structure and optical properties of a CsI single crystal, so we calculated the electronic structure and optical properties of the CsI crystal under high pressure after the geometrical structure of CsI had been optimized.

\subsection{Electronic structure}

After optimizing the crystal structure of the CsI single crystal under different high pressures, we calculated the electronic structure of the CsI crystal under a series of different high pressures. Fig. 2 shows the band structure of the CsI single crystal under different high pressures. The band gap of the CsI crystal with 0 pressure is $4.108 \mathrm{eV}$, and our result is a little bigger than that of other theoretical results ${ }^{8,11,24,25}$ due to the different exchange and correlation functions which have been used, but our result is closer to the experimental results ${ }^{26-28}$ when compared with their theoretical results. However, we still underestimate the band gap of the CsI crystal as compared to the experimental values. The DFT calculation always underestimates the band gap due to the LDA and GGA can not fully describe the exchange and correlation potentials among electrons as in the real system, but it has no remarkable effect on our research conclusions. The valence band of CsI consists of four parts, a high energy region (near the Fermi level), two medium energy regions (near $-7 \mathrm{eV}$ and $-11 \mathrm{eV}$ ) and a low energy region (near $-20 \mathrm{eV}$ ), and there is only one region in the conduction band of CsI. As the high pressure increases, the band gap of the CsI single crystal becomes smaller and smaller. This result is in agreement with the result in $\mathrm{Xu}^{\prime} \mathrm{s}^{17}$ research that high pressure can reduce the band gap of the CsI crystal. The luminescence spectrum of CsI is closely associated with its band structure, and comes from the de-excitation after the electrons that are in the CsI crystal are excited by X-rays or $\gamma$ rays. When electrons get enough energy from the rays, they can transit from the valence band to the conduction band and at the same time holes are produced in the valence band. When the excited electrons move back from the conduction band to the valence band, photons, whose energy is equal to the band gap, are produced. When a high pressure is put on the CsI crystal, the CsI crystal will produce more scintillation fluorescence photons (when it is exposed to the same amount of X-rays or $\gamma$ rays radiation) than that of CsI at $0 \mathrm{GPa}$. As we discussed above, high pressure can improve the detection performance of the CsI crystal, because the high pressure makes the crystal produce more visible photons than that of CsI at $0 \mathrm{GPa}$ when it is exposed to the ray radiation, and the best response energy of the photomultiplier is in the visible light range.

Fig. 3-5 show the total density of states (TDOS) of the CsI crystal under different high pressures, and the partial density of states (PDOS) of Cs atoms and I atoms, respectively. The TDOS of the CsI crystal is in good agreement with the band structure of CsI. In the valence band, the high energy region is formed by the $5 p$ orbit of the I atoms, the medium energy regions are formed by the $5 p$ orbit of the Cs atoms and the $5 \mathrm{~s}$ orbit of the I atoms, and the low energy region is formed by the $5 \mathrm{~s}$ orbit of the Cs atoms. The conduction band of CsI is formed by the $6 \mathrm{~s}$ and $5 \mathrm{p}$ orbit of the Cs atoms and the $5 \mathrm{~s}$ orbit of the I atoms. As the high pressure increases, the peaks in the TDOS and PDOS of CsI are broadening, and all the peaks move in the direction of decreasing energy. The peak values in the high energy region, medium energy regions (in the valence band) and the conduction band region decrease as the high pressure increases, but the peak value in the low energy region in the valence band increases with the increase of the high pressure.

We also investigated the effects of different high pressures on the atomic charge in the CsI single crystal, which is a good method to understand bonding behavior in a CsI crystal. The

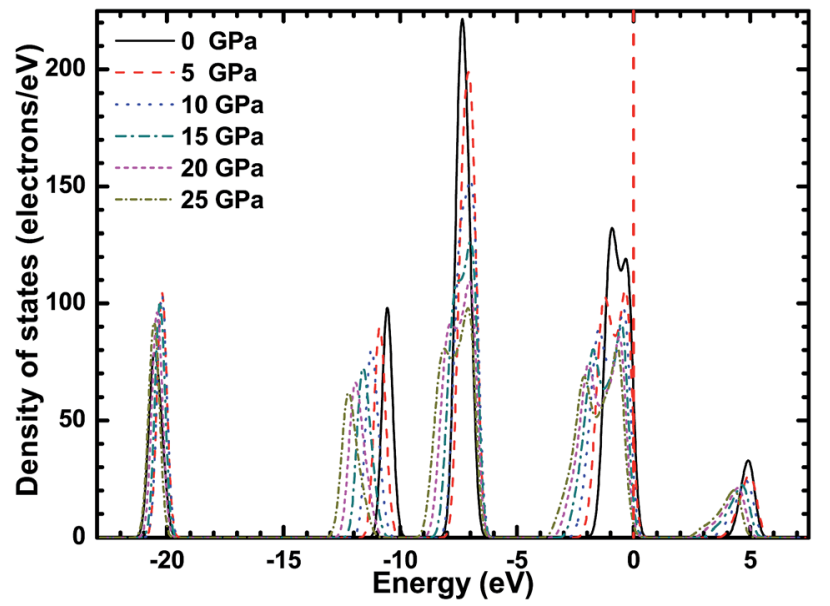

Fig. 2 The band structure of Csl single crystal under high pressures of 0 (a), 5 (b), 10 (c), 15 (d), 20 (e) and 25 (f) GPa.

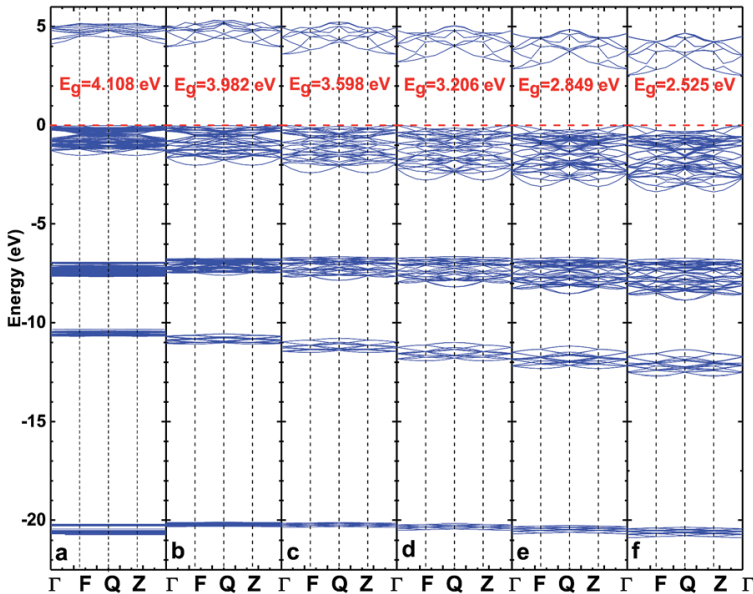

Fig. 3 The total density of states of the Csl single crystal under high pressure. 


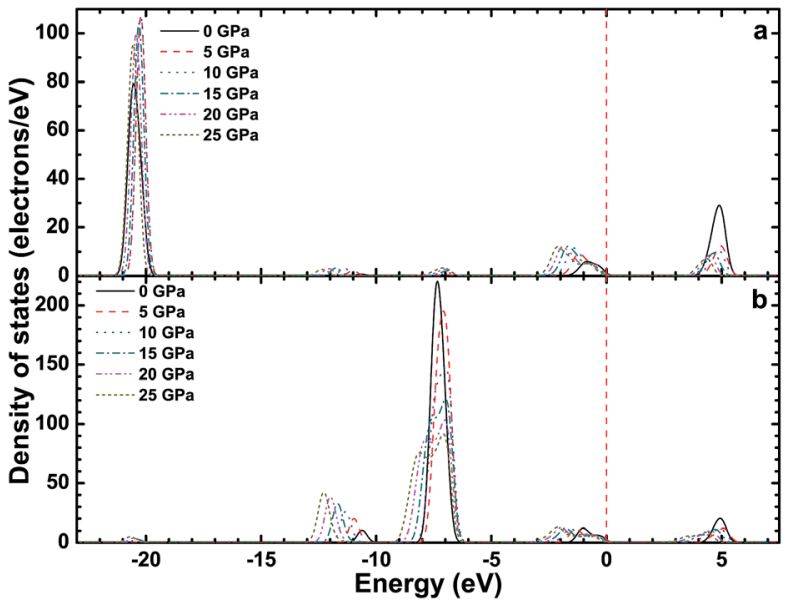

Fig. 4 The partial density of states of the sorbit (a) and p orbit (b) of Cs atoms in the Csl single crystal under high pressure.

atomic charge results are shown in Fig. 6 . The charge transfer from a Cs atom to an I atom is about 0.8 electrons and we concluded that the bonding behavior of CsI (at $0 \mathrm{GPa}$ ) is a combination of covalent and ionic nature. The result is consistent with our DOS calculation. The total atomic charge of an I atom is negative when the high pressure is less than $15 \mathrm{GPa}$, and the total atomic charge of an I atom is positive when the high pressure is greater than $15 \mathrm{GPa}$. By contrast, the atomic charge of a Cs atom is positive when the high pressure is less than $15 \mathrm{GPa}$, and the atomic charge of a Cs atom is negative when the high pressure is greater than $15 \mathrm{GPa}$. This result means that there is a charge transfer from a Cs atom to an I atom in CsI when the high pressure is less than $15 \mathrm{GPa}$, and that a charge transfer from an I atom to a Cs atom happens in CsI when the high pressure is greater than $15 \mathrm{GPa}$. Our result is in agreement with the result of Xu et al, ${ }^{17}$ that high pressure results in the peculiar reverse electron donation from I atoms to Cs atoms in a CsI crystal.

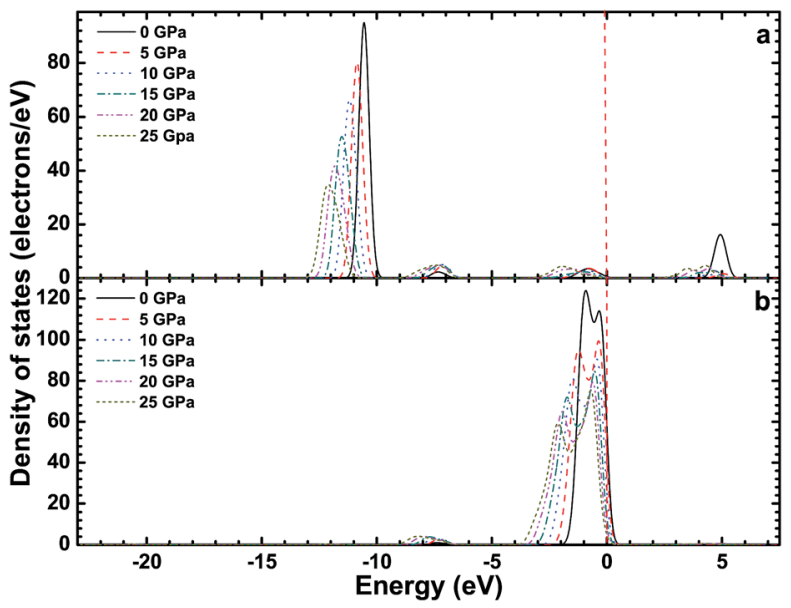

Fig. 5 The partial density of states of the s orbit (a) and p orbit (b) of I atoms in the Csl single crystal under high pressure.

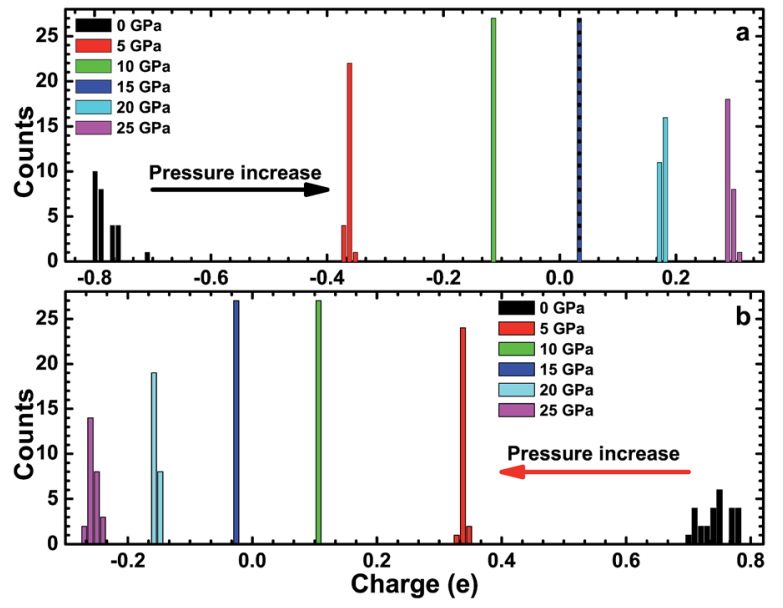

Fig. 6 The atomic charges of an I atom (a) and a Cs atom (b) in a CsI single crystal under high pressure.

\subsection{Optical properties}

Based on the electronic structure, the dielectric function of CsI under different high pressures was calculated, and $\varepsilon_{1}(\omega)$ and $\varepsilon_{2}(\omega)$ as a function of the photon energy are shown in Fig. 7. We can see from Fig. 7(a)that the intersection of the real part $\varepsilon_{1}(\omega)$ and vertical axis (at no external pressure) is about 1.3, this means that the static dielectric constant of the CsI crystal is about 1.3, and the static dielectric constant of the CsI crystal increases with the increase in external high pressure. In Fig. 7(b), there are two distinct peaks in the imaginary part of the dielectric function, the two peaks are at 6 and $12 \mathrm{eV}$. The first peak originates from the electron transition between the $5 p$ orbit (in the valence band) of an I atom and the $6 \mathrm{~s}$ orbit (in the conduction band) of a Cs atom, the second peak originates from electron transition between the $5 p$ orbit and the $6 \mathrm{~s}$ orbit of a Cs atom. When high pressure is applied to the CsI single crystal, the change to the first peak value is very weak, and while the second peak value becomes bigger and bigger, both peaks are

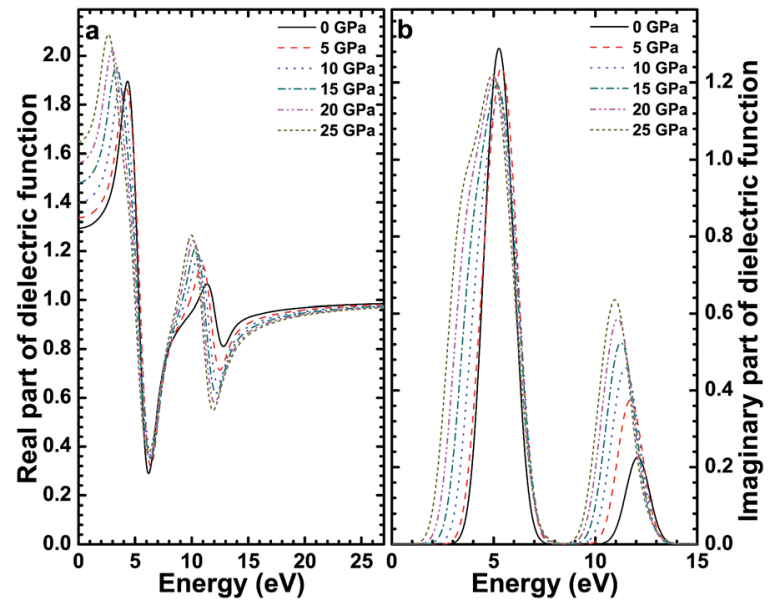

Fig. 7 The real part (a) and the imaginary part (b) of the dielectric function of the Csl single crystal under high pressure. 
redshifted. The reason for this result is that the band gap of CsI decreases with the increase of high pressure and the transition from the valence band to the conduction band needs less energy, so the redshift happens.

Fig. 8 shows the absorption spectrum of the CsI single crystal under different high pressures, and the results are identical to the imaginary part of the dielectric function of CsI. There are two peaks in the absorption spectrum of CsI, they are at 6 and $12 \mathrm{eV}$. The effect of high pressure on the first peak value is very weak, but the effect on the second peak is very obvious, it enhances the absorption of ultraviolet light by the CsI single crystal and this is useful for improving the detection efficiency of CsI crystals. The reason is that the band structure of the CsI crystal changes when a high pressure is put on it. The two peaks are expanded by the high pressure and both peaks are redshifted. The result is consistent with the conclusion from Makarenko. ${ }^{3}$ It is worth noting that CsI crystals are transparent to visible light (1.62-3.11 eV).

Fig. 9 shows the relationship between the refractive index of CsI and the energy of the photons under different high pressures. The static refractive index of the CsI single crystal is about 1.13 and the refractive index of CsI reaches its peak value of 1.4 when the photon's energy is equal to $4 \mathrm{eV}$. The refractive index of CsI increases with the increase in high pressure, and the static refractive index of CsI is 1.28 when the external high pressure is $25 \mathrm{GPa}$. The peak value increases as the high pressure increases, and both peaks move in the direction of decreasing energy. When the energy of the photon is bigger than $15 \mathrm{eV}$, the refractive index of the CsI crystal tends to be a constant value of 0.96 .

The reflectivity of the CsI crystal under different high pressures is shown in Fig. 10, and there are two peaks (at 6 and $12 \mathrm{eV}$ ) in the reflectivity of the CsI single crystal. The first peak broadens with the increase in external high pressure and the reflection of the CsI crystal on the low-energy photons has been enhanced by the external high pressure, this is useful for improving the ray detection efficiency of CsI crystals. The second peak value (at $12 \mathrm{eV}$ ) increases with the increase in

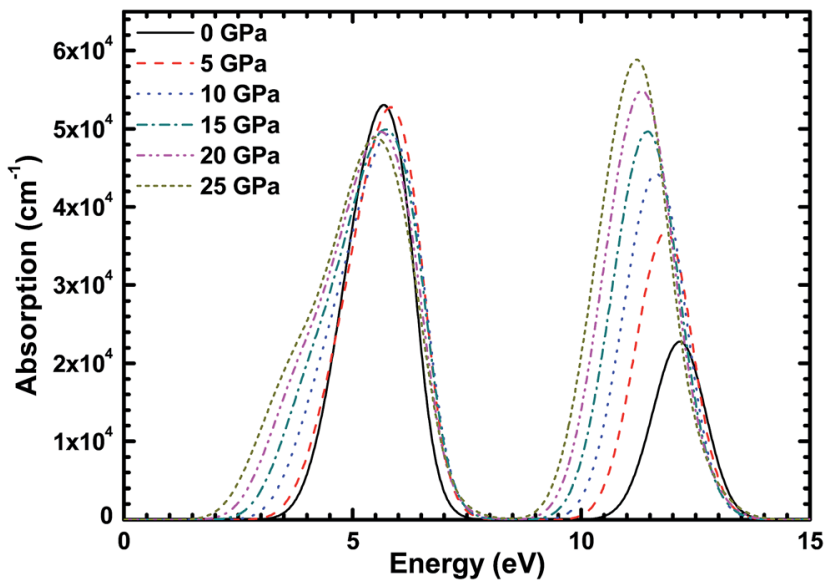

Fig. 8 The absorption spectrum of the Csl single crystal under high pressure.

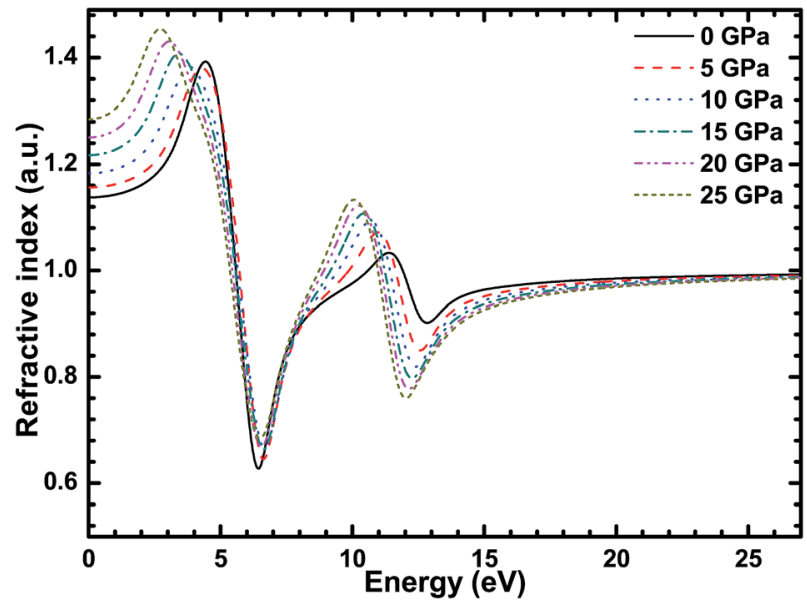

Fig. 9 The refractive index of the Csl single crystal under high pressure.

external pressure, and the peak is broadening. When the energy of the photon is bigger than $15 \mathrm{eV}$, the reflectivity of CsI tends to zero.

Fig. 11 shows the energy loss spectroscopy of CsI and it describes the energy loss when the photoelectrons go through a uniform dielectric. Its peak describes the resonant frequency of plasma, which corresponds to the sharp decrease in the reflectivity. It can be seen in Fig. 11 that there are two peaks in the loss function of the CsI crystal at no external pressure, and the two peaks are at 7 and $13 \mathrm{eV}$. The effect of high pressure on the first peak is very weak. As the external high pressure increases, the increase in the second peak value is very obvious, and a redshift occurs. The reason for this is that the inter-band transition in the CsI crystal under high pressure conditions becomes more active than that of the CsI crystal at 0 pressure.

From these results, we can see that high pressure can regulate and control the electronic structure and optical properties of a CsI scintillator very well and these changes will result in the

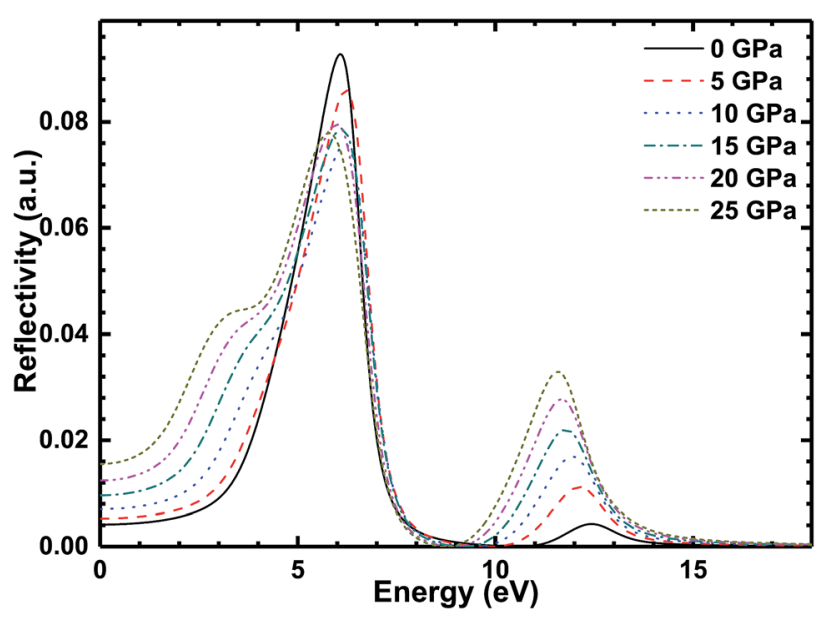

Fig. 10 The reflectivity of the Csl single crystal under high pressure. 


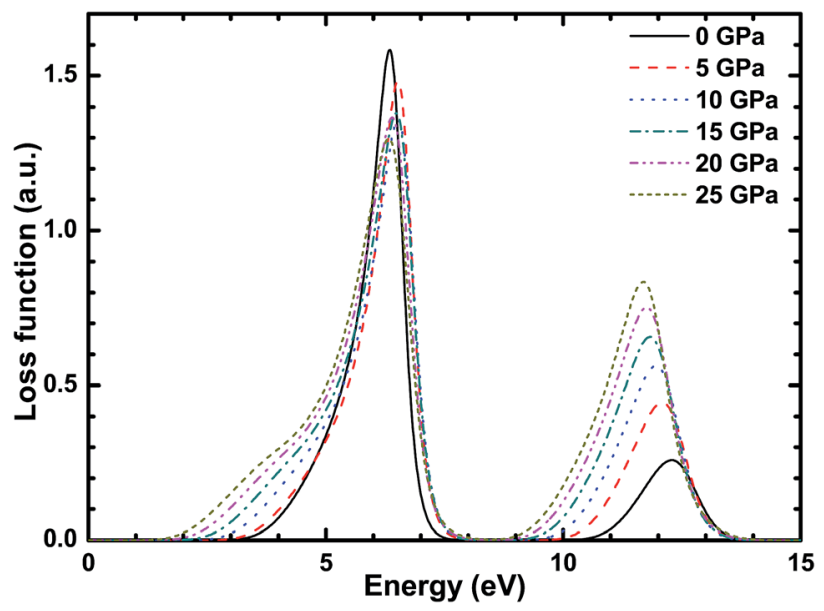

Fig. 11 The energy loss function of the Csl single crystal under high pressure.

CsI scintillator producing more visible photons at high pressure than that of the CsI scintillator at $0 \mathrm{GPa}$. This means that high pressure can enhance the detection efficiency of a CsI scintillator to X-rays and $\gamma$-rays.

\section{Conclusion}

Using a first-principles calculation method based on DFT, we systematically investigated the crystal structure, electronic structure and optical properties of a CsI single crystal under different high pressures. The results are as follows:

(1) The band gap of the CsI single crystal decreases with the increase in the external high pressure and the CsI crystal (under high pressure) will produce more visible photons compared to the CsI crystal at 0 GPa.

(2) Every part of the valence band becomes wider and wider as the high pressure increases and the peak values in the highenergy region, medium-energy region (in the valence band) and the conduction band decrease with the increase in high pressure. Every part, both the valence band and conduction band, moves in the direction of decreasing energy.

(3) There are two peaks in the optical parameters (such as the imaginary part of the dielectric function, absorption spectrum, refractive index, reflectivity and loss function) of the CsI crystal. For the imaginary part of the dielectric function, absorption spectrum and loss function, the effects of high pressure on the first peak value are very weak and on the second peak value they are more obvious. For the real part of the dielectric function, refractive index and reflectivity, the high pressure has an obvious effect on the first and second peak values. A redshift happens in all of these optical parameters.

All of these results indicate that the high pressure has an obvious effect on the electronic structure and optical properties of the CsI single crystal, and we can conclude that high pressure can improve the detection efficiency of CsI scintillators to X-rays and $\gamma$-rays. Our prediction in this paper might provide a new way to improve the detection performance of CsI scintillators.

\section{Conflicts of interest}

There are no conflicts to declare.

\section{Acknowledgements}

This work is supported by the Fundamental Research Funds of the Central Universities under grant No. 2017MS079.

\section{References}

1 E. Knittle, A. Rudy and R. Jeanloz, Phys. Rev. B, 1985, 31, 588. 2 L. J. Wang, L. C. Chen, F. Y. Li, H. C. Gu, L. Zhou and R. Z. Che, Chin. Phys. Lett., 1998, 15, 283-286.

3 I. N. Makarenko, A. F. Goncharov and S. M. Stishov, Phys. Rev. B, 1984, 29, 6018-6019.

4 H. K. Mao, R. J. Hemley, L. C. Chen, J. F. Shu, L. W. Finger and Y. Wu, Science, 1989, 246, 649-651.

5 M. I. Eremets, K. Shimizu, T. C. Kobayashi and K. Amaya, Science, 1998, 281, 1333.

6 R. M. Gaumé, S. Lam, M. Gascón, W. Setyawan, S. Curtarolo and R. S. Feigelson, Rev. Sci. Instrum., 2013, 84, 015109.

7 C. Li, B. Wang, R. Wang, H. Wang and Z. Zhu, Thin Solid Films, 2008, 516, 7894-7898.

8 R. M. Ribeiro, J. Coutinho, V. J. B. Torres, R. Jones, S. J. Sque, S. Öberg, M. J. Shaw and P. R. Briddon, Phys. Rev. B, 2006, 74, 035430.

9 Y. Kurosaki, L. Matsuoka, K. Yokoyama and A. Yokoyama, J. Chem. Phys., 2008, 128, 024301.

10 Y. Kurosaki and K. Yokoyama, Comput. Theor. Chem., 2012, 999, 239-245.

11 T. Liu, F. Yan, J. Chen and L. Liang, Chin. Opt. Lett., 2010, 8, 74-77.

12 X. Ying and Z. Ni, Comput. Mater. Sci., 2010, 48, 658-661.

13 M. B. Nardelli, S. Baroni and P. Giannozzi, Phys. Rev. B, 1995, 51, 8060.

14 S. Wei, C. Zhu, Q. Li, Y. Zhou, Q. Li and Y. Ma, Phys. Chem. Chem. Phys., 2014, 16, 17924-17929.

15 N. Ullah, G. Muraza, R. Khenata, A. H. Reshak, S. Naeem and M. N. Khalld, Int. J. Mod. Phys. B, 2014, 28, 1450047.

16 D. Baghmar, V. R. Mehto, N. K. Gaur and D. C. Gupta, AIP Conf. Proc., 2008, 299-302.

17 Y. Xu, S. T. John, O. R. Artem, T. Cui, H. Wang, Y. Ma and G. Zou, Phys. Rev. B, 2009, 79, 144110.

18 Z. Zhang, Q. Zhao, Y. Li and X. P. Ouyang, J. Korean Phys. Soc., 2016, 68, 1069-1074.

19 D. Y. Jiang, Z. Zhang, R. X. Liang, Z. H. Zhang, Y. Li, Q. Zhao and X. P. Ouyang, Nucl. Sci. Tech., 2017, 28, 32.

20 S. J. Clark, M. D. Segall, C. J. Pickard, P. J. Hasnip, M. I. J. Probert, K. Refson and M. C. Payne, Z. Kristallogr., 2005, 220, 567-570.

21 B. Hammer, L. B. Hansen and J. K. Nørskov, Phys. Rev. B, 1999, 59, 7414-7421.

22 R. D. L. Kronig, J. Opt. Soc. Am., 1926, 12, 547-556.

23 W. M. Haynes, Book Listing of CRC Handbook of Chemistry and Physics, American Chemical Society, 92nd edn, 2011.

24 L. W. Campbell and F. Gao, J. Lumin., 2013, 137, 121-131. 
25 S. A. Mian, M. Muzammil, G. Rahman and E. Ahmed, Mater. Sci.-Pol., 2017, 35, 197-210.

26 J. E. Eby, K. J. Teegarden and D. B. Dutton, Phys. Rev., 1959, 28 J. A. Bearden and A. F. Burr, Rev. Mod. Phys., 1967, 39, 125116, 1099-1105.
27 J. J. Hopfield and J. M. Worlock, Phys. Rev., 1965, 137, 14551464.

142. 